Psychotherapeut 2017 62:495-497

https://doi.org/10.1007/s00278-017-0238-0

Online publiziert: 27. Oktober 2017

๑) Springer Medizin Verlag GmbH 2017

CrossMark

\section{Christoph Herrmann-Lingen ${ }^{1} \cdot$ Christian Albus $^{2}$}

' Klinik für Psychosomatische Medizin und Psychotherapie und Deutsches Zentrum für HerzKreislaufforschung (DZHK), Universitätsmedizin Göttingen - Georg-August-Universität, Göttingen, Deutschland

${ }^{2}$ Klinik und Poliklinik für Psychosomatik und Psychotherapie, Universitätsklinikum Köln, Köln, Deutschland

\title{
Von Herzen
}

Zusammenhänge zwischen Funktion bzw. Erkrankungen des Herzens und psychischem Erleben finden sich nicht nur in zahlreichen Wendungen der Alltagssprache, sondern werden anekdotisch schon seit vorchristlicher Zeit immer wieder beschrieben. So heißt es bereits im ägyptischen Papyrus Ebers aus dem 16. vorchristlichen Jh.: „Wenn sein Herz trübselig ist und Trübnis geschmeckt hat, so ist sein Herz eingeengt, und Finsternis ist in seinem Leib in Folge von Zorn, was dergestalt wirkt, dass er sein Herz frisst" (Joachim 1890).

In den letzten 200 Jahren fand dann zunehmend eine wissenschaftliche Beschäftigung mit Herz-Seele-Wechselwirkungen statt, die insbesondere in den letzten 50 Jahren zu einer großen Fülle teilweise viel beachteter Publikationen geführt hat. Seit etwa 20 Jahren hat sich auf dieser Basis auch in Deutschland der interdisziplinäre Schwerpunktbereich „Psychokardiologie“ entwickelt. Meilensteine sind z.B. die Gründung der Arbeitsgruppe „Psychosomatik in der Kardiologie" des Deutschen Kollegiums für Psychosomatische Medizin (DKPM) im Jahr 1997, die „Statuskonferenz Psychokardiologie“ 1998-2002 sowie die Gründung der Arbeitsgruppe „Psychosoziale Kardiologie“ in der Deutschen Gesellschaft für Kardiologie - Herz- und Kreislaufforschung (DGK) 2002. In der Folge ist es zum deutlichen Wachstum psychokardiologischer Forschungsaktivitäten gekommen, in dessen Rahmen u. a. psychokardiologische Arbeitsgruppen und Professuren an mehreren deutschen Universitäten etabliert wurden. Auch eine der größten deutschen Psychotherapiestudien überhaupt, die multizentrische Studie
„Stepwise Psychotherapy Intervention for Reducing Risk in Coronary Artery Disease" (SPIRR-CAD; Albus et al. 2011; Herrmann-Lingen et al. 2016), wurde auf dieser Grundlage durchgeführt. Psychokardiologische Inhalte konnten zunehmend in Leitlinien bzw. Positionspapieren (z. B. Gohlke et al. 2013; Bundesärztekammer 2016; Ladwig et al. 2013) sowie in Aus-, Fortund Weiterbildungscurricula verankert werden. Psychokardiologische Versorgungsangebote werden heute mehr und mehr an Krankenhäusern und Rehabilitationskliniken etabliert. Regelmäßige interdisziplinäre Fortbildungskurse in psychokardiologischer Grundversorgung (unter dem Dach der DGK) dienen der Förderung des Dialogs von Psychologen/Psychotherapeuten, psychosomatisch tätigen Ärzten und Kardiologen mit dem Ziel, die ganzheitliche somatische und psychotherapeutische Behandlung von Herzpatienten im stationären und im ambulanten Bereich $\mathrm{zu}$ verbessern (Herrmann-Lingen et al. 2014). Mit wachsender Etablierung der Psychokardiologie werden auch vermehrt psychotherapeutische Angebote für Patienten mit Herzerkrankungen oder herzbezogener Symptomatik nachgefragt. HerzKreislauf-Erkrankungen sind nicht nur häufig, sondern werden auch in relevantem Ausmaß durch psychische, soziale und Verhaltensfaktoren mitverursacht und gehen oft mit psychischer Komorbidität einher. Insofern verwundert es nicht, dass immer häufiger Indikationen zur psychotherapeutischen Mitbehandlung von Herzpatienten gestellt werden.

Die Zeitschrift Psychotherapeut hat diesem Bedarf bereits in der Vergangenheit immer wieder durch Einzelbeiträge
Rechnung getragen. Im vorliegenden Heft findet sich nun erstmals ein eigener Themenschwerpunkt Psychokardiologie, in dem sowohl die psychischen Folgen kardialer Erkrankungen und ihre Behandlung als auch die Bedeutung früher Traumatisierungen und maladaptiver Verhaltensweisen für die Entstehung von Herzkrankheiten thematisiert werden. Grundtenor ist, dass Herzerkrankungen, als psychosomatische Krankheitsbilder verstanden, sinnvollerweise weder ausschließlich somatisch noch ausschließlich psychotherapeutisch behandelt werden können. Auch ein unkoordiniertes Nebeneinander körpermedizinischer und psychotherapeutischer Behandlungen ergibt oft unbefriedigende Ergebnisse. Insofern stellt das vorliegende Heft ebenfalls einen Aufruf $\mathrm{zu}$ intensiverem Austausch und Kooperation zwischen Psychotherapeuten und somatisch behandelnden Ärzten dar.

Das Thema des Hefts wird in folgenden 5 Beiträgen aus unterschiedlichen Perspektiven beleuchtet:

Herzerkrankungen stellen vielfach in der Fantasie, teilweise aber auch real lebensbedrohliche Extremsituationen dar. Dabei kann Todesangst sowohl bei einem akuten Herzinfarkt als auch im Rahmen einer somatoformen Störung oder Angststörung auftreten. Gerade bei Patienten mit manifester Herzerkrankung lässt sich beides nicht immer eindeutig differenzieren. Koronarpatienten benötigen einerseits eine realitätsadäquate Angst als wichtigen Motivator zu Selbstfürsorge und Inanspruchnahme ärztlicher Hilfe im Notfall; gerade ein Übermaß an Verleugnung wird ja hier häufig zum klinischen Problem. Andererseits kann die Angst über das adaptive Maß hinaus- 
gehen und als neurotische Angst bzw. „pathologische Realangst“ (Linden et al. 2008) eine somatoforme Überlagerung der Organerkrankung bedingen. Oft kommt es dann zu ausgeprägtem Kontroll- und Sicherheitsverhalten sowie häufiger Inanspruchnahme ärztlicher Rückversicherung und Diagnostik. Der Beitrag von Michal et al. widmet sich vor diesem Hintergrund der Bedeutung von Todesangst bei Patienten mit Brustschmerzen. Er diskutiert die Prävalenz und die prognostische Bedeutung der Todesangst sowie ihre funktionalen und auch dysfunktionalen Aspekte und lädt zu einem affektfokussierten Zugang ein, den er an einem Patientenbeispiel verdeutlicht. Dabei veranschaulicht er den Teufelskreis aus psychodynamischen Faktoren, Brustschmerz und Angsterleben am Beispiel eines Patienten nach durchgemachtem Herzinfarkt.

Auch im Beitrag von Peregrinova und Hamann steht die existenzielle Bedrohung von Herzpatienten im Mittelpunkt, indem die Folgen einer Behandlung mithilfe implantierter Defibrillatoren (ICD) fokussiert werden. Die ICD können einerseits Leben retten, indem sie potenziell tödliche Herzrhythmusstörungen durch elektrische Überstimulation oder Elektroschocks beenden, andererseits stellt die Kombination aus zugrunde liegender Herzkrankheit, Rhythmusstörungen und ICD-Schocks für die Patienten eine erhebliche psychische Belastung dar. Nicht selten erfüllt diese die Merkmale eines psychischen Traumas und kann Traumafolgestörungen nach sich ziehen. Auf der Basis eines Literaturüberblicks und reichhaltiger klinischer Erfahrung wird ein Behandlungskonzept für Patienten vorgestellt, die nach dem Erleben von Defibrillatorschocks posttraumatische Symptome entwickelt haben. In diesem Konzept spielt die Behandlung mittels „Eye Movement Desensitization and Reprocessing" (EMDR) eine zentrale Rolle. An Fallbeispielen wird das psychotherapeutische Vorgehen, wie es in der Klinik der Autorinnen praktiziert wird, anschaulich erläutert, wobei teilweise schon mit wenigen Sitzungen erhebliche symptomatische Besserungen erzielt werden können.
Die Übersichtsarbeit von Waller befasst sich demgegenüber mit frühen biografischen Traumatisierungen und deren Effekten auf das Herz-Kreislauf-System. Hier ist die Herzerkrankung also nicht Auslöser traumatischen Erlebens, sondern eine Traumatisierung trägt langfristig zu deren Entwicklung bei. Waller führt aus, dass sich die "familiäre Belastung" als etablierter kardiovaskulärer Risikofaktor nicht nur genetisch verstehen lässt, sondern dass das Risiko von Herzkrankheiten auch über epigenetische Mechanismen erhöht wird. Gleichzeitig weist sie darauf hin, dass ein moderates Ausmaß von Stressbelastungen in der frühen Lebensphase möglicherweise günstige gesundheitliche Folgen haben kann. Schließlich geht sie auf die transgenerationale Weitergabe früher Traumatisierungen ein, für die sie epigenetische und Verhaltensmechanismen identifiziert, über diefrüh traumatisierte Mütter ein erhöhtes Erkrankungsrisiko, unter bestimmten Umständen aber möglicherweise auch eine besondere Resilienz an ihre Kinder weitergeben könnten.

Fangauf et al. greifen den Zusammenhang zwischen Gesundheitsverhalten und Verlauf der koronaren Herzkrankheit auf. Insbesondere psychisch belasteten Herzpatienten gelingt es häufig nicht, ihr Leben an die Anforderungen der Erkrankung anzupassen und gesundheitsschädliche Verhaltensweisen zugunsten besserer Selbstfürsorge aufzugeben. Deshalb ist es oft trotz kardiologischer Behandlung nicht möglich, ihre kardiovaskulären Risikofaktoren ausreichend zu kontrollieren. Auch rein psychotherapeutische Behandlungsansätze scheinen hier wenig zu bewirken, da eine Besserung von Ängsten oder Depressivität nicht automatisch auch zu besserem Gesundheitsverhalten führt. Es wird daher das aus den USA stammende Konzept der "collaborative care“ bzw. TeamCare vorgestellt, in dem speziell trainierte und regelmäßig interdisziplinär supervidierte nichtärztliche Behandlungsassistenten die Patienten längerfristig beim Leben mit der Herzerkrankung, bei der Bewältigung psychischer Begleitsymptome und bei der Anpassung des Lebensstils unterstützen. Diese kooperieren eng mit anderen Ver- sorgern wie Hausärzten, Kardiologen und ggf. auch Psychotherapeuten und koordinieren und unterstützen deren Bemühungen. Anhand von Erfahrungen aus einer eigenen Pilotstudie wird das Vorgehen erläutert und an Beispielen von Patienten und ihrer Behandlungsverläufe verdeutlicht.

Während sich die bisherigen Beiträge im Wesentlichen mit psychosomatischen und psychotherapeutischen Aspekten von Herzerkrankungen beschäftigen, geht es im Beitrag von Schmidt und Martin zum Herzraten-Biofeedback um eine Methode, die sich unabhängig vom Vorliegen einer Herzerkrankung der Registrierung und der Beeinflussung der Herzschlagfolge bedient, um hierüber Einfluss auf das autonome Nervensystem zu nehmen. Damit sollen im Interesse einer allgemeinen Gesundheitsförderung die vegetative Balance verbessert sowie körperliche und psychische Symptome reduziert werden. Die Autorinnen stellen sowohl die dieser Methode zugrunde liegenden theoretischen Annahmen als auch empirische Befunde für die Wirksamkeit des Verfahrens dar. Sie präsentieren eine systematische Literaturübersicht existierender Wirksamkeitsstudien, die nahelegt, dass das Herzraten-Biofeedback zumindest gegenüber inaktiven Kontrollbedingungen günstige physiologische und psychologische Effekte haben kann.

Wir hoffen, Ihnen mit diesem Schwerpunktheft viele Anregungen zur vertieften Auseinandersetzung mit dem Gebiet der Psychokardiologie zu geben und Ihre Neugier auf ein integriertes psychotherapeutisches Arbeiten mit Herzpatienten zu wecken. Neben dem besseren Verständnis der somatischen Krankheitsprozesse, ihrer psychophysiologischen Hintergründe sowie therapeutischer Konzepte erfordert das psychotherapeutische Arbeiten mit Herzpatienten freilich auch die Auseinandersetzung mit eigenen Todesängsten sowie Gefühlen von Hilflosigkeit angesichts der oft fortschreitenden und noch immer häufig zum Tod führenden körperlichen Grunderkrankung. Insofern möchten wir dazu einladen, sich der Thematik zu nähern, auch im Rahmen von Selbsterfahrung, Super- oder Intervisionen oder 
den Kursen der psychokardiologischen Grundversorgung der DGK, die sich explizit auch an psychotherapeutisch tätige Kolleginnen und Kollegen wenden.

\section{Korrespondenzadresse}

Prof. Dr. C. Herrmann-Lingen

Klinik für Psychosomatische Medizin und Psychotherapie und Deutsches Zentrum für Herz-Kreislaufforschung (DZHK), Universitätsmedizin Göttingen - GeorgAugust-Universität Von-Siebold-Str. 5, 37099 Göttingen, Deutschland cherrma@gwdg.de

Interessenkonflikt. C. Hermann-Lingen weist auf folgende Beziehungen hin: Honorare für Publikationen und Vorträge zu psychokardiologischen Themen; Universitätsmedizin Göttingen: leistungsabhängige Entgeltbestandteile für psychokardiologische Behandlungen von Privatpatienten. C. Albus weist auf folgende Beziehungen hin: Honorare für Publikationen und Vorträge zu psychokardiologischen Themen.

\section{Literatur}

Albus C, Beutel M, Deter HC, Fritzsche K, Hellmich M, Jordan J, Jünger J, Krauth C, Ladwig KH, Michal M, Mueck-Weymann M, Petrowski K, Pieske B, Ronel J, Söllner W, Waller C, Weber C, HerrmannLingen C (2011) A stepwise psychotherapy intervention for reducing risk in coronary artery disease (SPIRR-CAD) - rationale and design of a multicenter, randomised trial in depressed patients with CAD. J Psychosom Res 71:215-222

Bundesärztekammer (BÄK) (2016) Kassenärztliche Bundesvereinigung (KBV), Arbeitsgemeinschaft der Wissenschaftlichen Medizinischen Fachgesellschaften (AWMF). Nationale VersorgungsLeitlinie Chronische KHK - Langfassung, 4.Auflage.https://doi.org/10.6101/AZQ/000267

Gohlke H, Albus C, Bönner G, Darius H, Eckert S, Gohlke-Bärwolf C, Gysan D, Hahmann H, Halle N, Hambrecht R, Mathes P, Predel HG, Sauer G, von Schacky C, Schuler G, Siegrist J, Thiery J, Tschöpe D, Völler H, Wirth A (2013) Empfehlungen der Projektgruppe Prävention der DGK zur risikoadjustierten Prävention von Herz-Kreislauferkrankungen. Teil 4: Thrombozytenfunktionshemmer, Hormonersatztherapie, Verhaltensänderung und psychosoziale Risikofaktoren. Kardiologe 7:297-306

Herrmann-Lingen C, Albus C, Titscher G (Hrsg) (2014) Psychokardiologie. Ein Praxisleitfaden für Ärzte und Psychologen, 2. Aufl. Deutscher ÄrzteVerlag, Köln

Joachim H (1890) Papyros Ebers. Das älteste Buch über Heilkunde Bd. 2. Reimer, Berlin (Aus dem Aegyptischen zum ersten Mal vollständig übersetzt)

Ladwig KH, Lederbogen F, Albus C, Angermann C, Borggrefe $M$, Fischer $D$, Fritzsche $K$, Haass $M$, Jordan J, Jünger J, Kindermann I, Köllner V, Kuhn B, Scherer M, Seyfarth M, Völler H, Waller C, Herrmann-Lingen C (2013) Positionspapier zur Bedeutung von psychosozialen Faktoren in der Kardiologie. Update 2013. Kardiologe 7:7-27
Linden M, Dirks S, Glatz J (2008) Die „pathologische Realangst" am Beispiel kardiovaskulärer Erkrankungen. Psychosom Konsiliarpsychiatr 2:248-254

Herrmann-Lingen C, Beutel ME, Bosbach A, Deter HC, Fritzsche K, Hellmich M, Jordan J, Jünger J, Ladwig KH, Michal M, Petrowski K, Pieske B, Ronel J, Söllner W, Stöhr A, Weber C, de Zwaan M, Albus C, SPIRR-CAD Study Group (2016) A stepwise psychotherapy intervention for reducing risk in coronary artery disease (SPIRR-CAD): results of an observer-blinded, multicenter, randomized trial in depressed patients with coronary artery disease.Psychosom Med 78:704-715

\section{Schwerpunktthemen}

Das Herausgebergremium der Zeitschrift Psychotherapeut lädt Autorinnen und Autoren ein, an den geplanten Schwerpunkten mitzuarbeiten und geeignete Manuskripte einzureichen. Diese werden dem üblichen Reviewverfahren unterzogen. Darüber hinaus freuen wir uns über die Zusendung freier (unaufgeforderter) Originalia zu selbstgewählten Themen.

Bitte schicken Sie Ihren Beitrag an die Redaktion:

Regine.Karcher-Reiners@springer.com

Die Schwerpunktplanung ist vorläufig und kann kurzfristig umdisponiert werden.

Heft 2/2018: Angst, Ressentiment, Hoffnung. Hass, Fanatismus, Versöhnung. Lindauer Psychotherapiewochen 2017

Heft 3/2018: Angst (Deadline 30.12.2017)

Heft 4/2018: Digitalisierung in der Psychotherapie (Deadline 30.02.2018)

Heft 5/2018: Sexualität und sexuelle Störungen (Deadline 30.04.2018)

Heft 6/2018: Studium zur Approbation (Deadline 30.06.2018) 